\title{
Von Bildern zu Bühnen: Die Geoglyphen von Palpa und Nasca (Süd-Peru) in ihrem räumlichen und sozialen Kontext
}

This paper deals with geoglyphs from Palpa and Nasca (Southern Peru) in a spatial and social context. In one of the first phases, the Paracas Period, geoglyphs were predominately situated on slopes, and their repertoire of motifs (mainly anthropomorphic beings) was taken from other media. These depictions must have been constructed in connection with activities that took place at a certain distance and that cannot be fully ascertained. In the subsequent Nasca Period the populace went on to construct enormous geoglyphs in the form of lines or other geometric patterns on the desert-like plains between river valleys. These geoglyphs henceforth came to serve as stages for numerous ritual activities (as shown by offering deposits). These rites were presumably connected with the increasing aridisation of the climate and with prayers for water and fertility. The important thing here was no longer so much the picture, the geoglyph, but rather the activities performed upon them, activities in which other media now came to assume the role of image bearer.

\section{Einführung}

Die Geoglyphen zwischen Palpa und Nasca an der Südküste Perus, auch als >NascaLinien< bekannt, sind ein in Art und Umfang einzigartiges Beispiel prähistorischer Landschaftsgestaltung. Mehr als $500 \mathrm{~km}^{2}$ der Steinwüste des Nasca-Beckens am Fuße der Anden (Abb. 1) wurden zwischen ca. 350 v. Chr. und 700 n. Chr. durch die damaligen Bewohner der Region mit Bodenzeichnungen überzogen und damit nach ihren kulturellen Konzepten und Bedürfnissen großflächig und dauerhaft umgestaltet. Die verwendete Technik der Steinumlagerung war dabei denkbar einfach; die Lage in der Wüste, wo natürliche Erosion und sonstige menschliche Nutzung nur eine geringe Rolle spielen, trug zur weitgehenden Erhaltung dieser Bodendenkmäler bei. Über Funktion und Bedeutung der Geoglyphen sind uns keinerlei Informationen überliefert. Aus vorspanischer Zeit existieren für ganz Südamerika keine Schriftquellen; zudem wurde die Geoglyphentradition bereits viele Jahrhunderte bevor spanische und einheimische Chronisten nach der Conquista (ab 1532 n. Chr.) kulturelle und religiöse Eigenheiten des Andenraumes beschrieben aufgegeben.

Somit konnte nach der Wiederentdeckung der Geoglyphen durch Wissenschaftler in den 1920er Jahren zunächst allein ihre Gestalt Anhaltspunkte zu ersten Interpretationen liefern. Dazu trug der damals aufkommende Flugverkehr entscheidend bei. Erst aus der Vogelperspektive wurden Ausmaß und Bedeutung des Geoglyphen-Phänomens erkannt und so bestimmte die Sicht von oben, die einen Überblick über die Geoglyphen erlaubte, viele Deutungsversuche ${ }^{1}$. Aus der Vogelperspektive erscheinen die großflächig

$1 \quad$ Horkheimer 1947; Kosok - Reiche 1947. 
über den Wüstenboden ausgebreiteten Geoglyphen wie auf eine Wandtafel gemalt. Es ist daher nicht verwunderlich, dass die Geoglyphen häufig als Bilder aufgefasst und gedeutet werden. Dies liegt besonders bei einigen figürlichen Geoglyphen nahe, die in der Tat leicht als Abbildungen von Tieren zu erkennen sind. Dazu zählen so bekannte Figuren wie Kondor, Hund, Spinne, Kolibri, Affe, Eidechse und Pelikan, die meisten davon auf den Pampas von Nasca zwischen Río Ingenio und Río Nasca ${ }^{2}$. Diese figürlichen Darstellungen prägen auch heute noch das Bild der Geoglyphen in der Öffentlichkeit, da sie das wichtigste Ziel der täglichen touristischen Flüge über die Pampas von Nasca sind.

Eine simple Interpretation der Geoglyphen als Abbildung natürlicher Objekte greift jedoch deutlich zu kurz. Das wird schon allein dadurch klar, dass die figürlichen Geoglyphen trotz ihres hohen Bekanntheitsgrades nur einen Bruchteil des Corpus ausmachen. In diesem Corpus dominieren stattdessen geometrische Geoglyphen, die aus Linien und Flächen in verschiedenen Größen und Formen gebildet werden. Diese Bodenzeichnungen können nicht einfach als Bilder angesprochen werden, da sich ihre Formensprache stark von der vorspanischen Ikonographie der Region unterscheidet, die von Keramik und Textilien bestens bekannt ist. Zudem entspricht die Vogelperspektive nicht der Perspektive der Erbauer und Nutzer, die die Geoglyphen am Boden konzipierten, anlegten und erlebten. Zwar sind alle Geoglyphen am Boden als solche erkennbar, doch können viele von ihnen, seien es figürliche Darstellungen oder geometrische Kombinationen, nicht gänzlich überblickt und daher auch nicht wie Bilder betrachtet werden.

Nachdem ab den 1940er Jahren die Geoglyphen sukzessiv auch am Boden kartiert und untersucht wurden, rückten die zahlreichen Linien, Trapeze und anderen geometrischen Geoglyphen und damit auch ein anderer Deutungsansatz in den Mittelpunkt der Diskussion ${ }^{3}$. Die Geoglyphen wurden nun als ein gigantischer astronomischer Kalender verstanden ${ }^{4}$. Man nahm an, dass Linien und Trapeze auf Punkte am Horizont wiesen, an denen zu kalendarisch bedeutsamen Daten die Sonne oder andere Himmelskörper aufbzw. untergingen oder ihren Zenit durchschritten. Diese astronomische Hypothese hatte den Vorzug, dass sie von der Bodenperspektive ausging und - in der Rolle des Betrachters auch die Erbauer und Nutzer der Geoglyphen einbezog. Der damalige Kenntnisstand über die Kulturgeschichte der Nasca-Region blieb jedoch weitgehend unberücksichtigt. Verschiedene Untersuchungen in den folgenden Jahrzehnten ergaben dann auch, dass die Orientierung der großen Mehrheit der geometrischen Geoglyphen nicht mit astronomischen Konstellationen erklärt werden kann ${ }^{5}$.

Aus der Sicht jüngerer Ansätze der Landschaftsarchäologie ${ }^{6}$ weisen bisherige Deutungsversuche das Manko auf, dass sie die soziale Dimension der Anlage und Nutzung

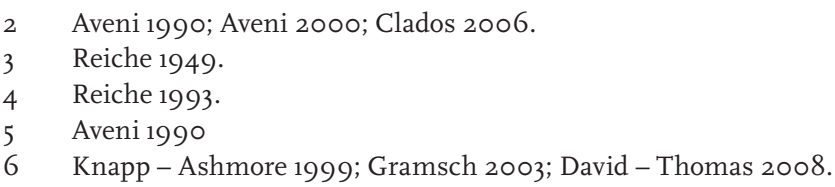


der Geoglyphen vernachlässigen. Zum einen sind die damaligen Bewohner der Region als handelnde Akteure stärker in den Blick zu nehmen. Sowohl der erhebliche Arbeitsaufwand, der zur Anlage der Geoglyphen notwendig war, als auch die konkret umgesetzten Formen und Merkmale der Geoglyphen mussten kulturell eingebettet und sozial legitimiert sein. Die auf den Geoglyphen anzunehmenden Aktivitäten, die sich nicht auf reine Beobachtung beschränkt haben dürften, sind zu rekonstruieren und auf ihre soziale und kulturelle Rolle hin zu untersuchen. In der Tat fanden schon die frühen Erforscher der Geoglyphen zahlreiche Hinweise auf solche Aktivitäten, ohne sie jedoch stärker in ihre Interpretationen einzubeziehen ${ }^{7}$. Zum anderen definieren Akteure auch soziale Räume, Aktivitäten auch Handlungsräume in der Wüste ${ }^{8}$. Es ist also von einem erweiterten Raumkonzept auszugehen, das nicht nur durch natürliche, sondern auch durch soziale und kulturelle Parameter definiert wird. In diesem Sinne gibt das Thema »Bild - Raum Handlung« der Berliner Topoi-Tagung einen geeigneten Rahmen für eine zeitgemäße Interpretation der Geoglyphen vor.

Eine gute Grundlage, um solchen Fragen nachzugehen, boten die zwischen 1997 und 2004 vom Archäologischen Projekt Nasca-Palpa durchgeführten archäologischen Untersuchungen der Geoglyphen von Palpa im nördlichen Teil des Nasca-Beckens 9 . Im Folgenden werden zunächst das Nasca-Becken selbst, sodann die frühen biomorphen Geoglyphen der Paracas-Zeit und schließlich die geometrischen Geoglyphen der Nasca-Zeit in der Umgebung von Palpa vorgestellt und in ihrem räumlichen und sozialen Kontext interpretiert. Die zugrundeliegenden archäologischen Daten wurden bereits an anderer Stelle vorgelegt ${ }^{10}$.

\section{Das Nasca-Becken}

Das Nasca-Becken in Süd-Peru (Abb. 1) befindet sich in einem nur ca. 50-70 km breiten Wüstenstreifen zwischen der Pazifikküste im Südwesten und der Andenkordillere im Nordosten. Obwohl in der Nähe des Meeres gelegen, ist es von diesem durch eine

\footnotetext{
7 Ausnahme: Horkheimer 1947.

8 Vgl. Llobera 1996.

9 Das Archäologische Projekt Nasca-Palpa wurde 1997 initiiert und mit verschiedenen Schwerpunkten bis heute fortgeführt. Es wird geleitet von Markus Reindel (Bonn) und Johny Isla (Lima). Beteiligt sind u. a. das DAI, die ETH Zürich, die Universität Heidelberg und weitere Institutionen (s. den Überblick in Reindel - Wagner 2009, auf deren Band auch die im Text genannten Datierungen beruhen). Die Finanzierung erfolgte zunächst durch die SLSA, Zürich, sodann durch das BMBF, Bonn; weitere Beiträge zu den hier beschriebenen Arbeiten kamen vom DAI, der ETH und der Universität Zürich. Für die enge und fruchtbare Zusammenarbeit bei der Erforschung der Geoglyphen von Palpa danke ich den Projektleitern Markus Reindel und Johny Isla, den Betreuern meiner Dissertation, Philippe Della Casa und Armin Grün, sowie meinen Kollegen Martin Sauerbier, Sabine Beutner, Juan Carlos De La Torre, Alfredo Bautista und Alejandra Figueroa.

10 Lambers 2006; Reindel et al. 2006.
} 
niedrige Küstenkordillere getrennt und befindet sich mit 300-600 $\mathrm{m}$ deutlich über dem Meeresspiegel, so dass es sich eigentlich nicht um eine Küstengegend handelt. Wie in fast ganz Peru und in weiten Teilen Nordchiles herrschen im schmalen Landstreifen zwischen Küste und Anden extrem aride Bedingungen. Saisonale Regenfälle in den Anden speisen jedoch eine Reihe von Flüssen, die die Wüste durchschneiden und in den Pazifik entwässern. In der Nasca-Region fließen diese vor der Küstenkordillere zunächst im Río Grande zusammen, bevor dieser Fluss als einziger das Meer erreicht. So ergibt sich eine an der peruanischen Küste einzigartige Beckensituation.

Dieses Becken wurde bis vor ca. 45.000 Jahren mit unterschiedlich groben Sedimenten angefüllt, die von der Andenwestabdachung aberodierten und am Andenfuß flache Fußfächen und Schwemmkegel ausbildeten ${ }^{11}$. In diese mächtigen Ablagerungen schnitten sich in der Folgezeit die Flüsse ein, so dass die heutigen Flusstäler deutlich unter dem Niveau der Fußflächen liegen. Auf diese Weise entstand am Andenfuß die charakteristische Landschaft aus wüstenhaften, annähernd flachen Fußflächen (pampas), die von Trockentälern (quebradas) und fruchtbaren Flussoasen unterbrochen werden.

Die Flusstäler mit ihren saisonal Wasser führenden Flüssen und fruchtbaren Böden stellten schon immer die Lebensgrundlage für die ansässige Bevölkerung dar und tun dies bis heute. In der Paracas-Zeit (800-200 v. Chr.) und der Nasca-Zeit (120 v. Chr. - 620 n. Chr.), um die es im Folgenden geht, wurde jedoch durch die Anlage von Geoglyphen auch die Wüste in die kulturelle Domäne der Gesellschaft einbezogen. Die Geoglyphen konnten leicht angelegt werden, da sich an der Oberfläche der Ablagerungen, die die Beckenfüllung bildeten, durch Ausblasen des Feinsedimentes ein dichtes Wüstenpflaster aus rotbraun oxidierten Steinen auf einer Schicht helleren und lockereren Sedimentes gebildet hatte ${ }^{12}$. Zur Anlage von Bodenzeichnungen mussten lediglich die dunklen Steine aufgesammelt und an anderer Stelle deponiert werden, um einen starken Kontrast zwischen dem originalen Wüstenpflaster und dem exponierten helleren Sediment zu erzielen. Wo das freigelegte Sediment nicht durch häufige Begehung stark kompaktiert war, wurde es zwar durch Winderosion nach und nach beeinträchtigt, so dass die Stärke der Erosionsspuren einen ersten Hinweis auf das relative Alter der Geoglyphen geben kann. Aber insgesamt blieben die Geoglyphen bemerkenswert gut erhalten, da trotz diverser Klimaschwankungen die Kernzone der Geoglyhen seit der Nasca-Zeit weitgehend frei von Niederschlägen blieb ${ }^{13}$. Ihre größte Bedrohung stellt heute neben den sehr seltenen Regenfällen die Nutzung des Geländes durch den Menschen dar.

Des Weiteren ist der Naturraum des Nasca-Beckens von Elementen in der Umgebung geprägt, die für sein Verständnis wichtig sind ${ }^{14}$. Der Horizont wird durch die das Becken

\footnotetext{
$11 \quad$ Mächtle 2007.

12 Eitel et al. 2005.

13 Eitel - Mächtle 2009.

14 Mächtle 2007.
} 
Abb. 1 | Das Nasca-Becken in Süd-Peru zwischen Pazifik und Anden.

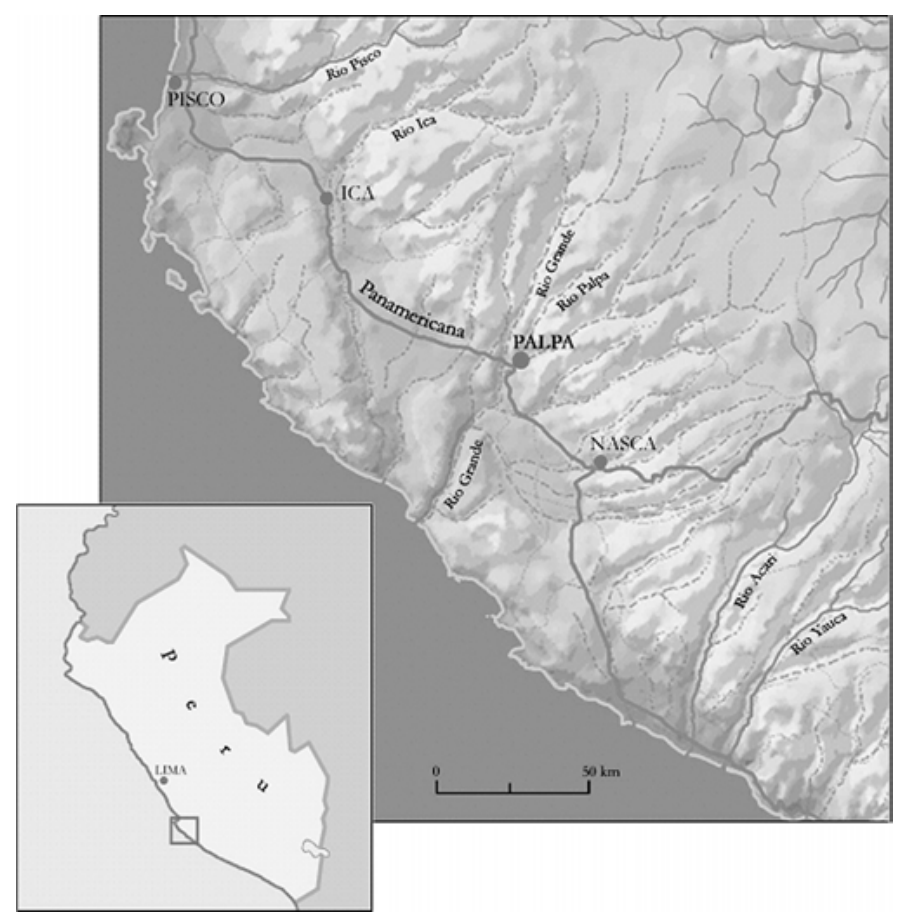

bildenden Hügel und Berge in allen Himmelsrichtungen definiert, vor allem durch die massive, die Pampas überragende Andenkordillere im Nordosten. Aus dieser erreichen die so wichtigen Flüsse das Becken; sie bieten zudem schon in kurzer Entfernung die Täler hinauf deutlich feuchtere Bedingungen und damit ergänzende Ressourcen. Das Meer im Südwesten ist zwar nicht sichtbar, bestimmt aber mit dem vorherrschenden Wind aus dieser Richtung, der oft Wolken, manchmal auch Sandstürme mit sich bringt, dennoch den Charakter des Naturraumes mit. Auch das Meer bietet in erreichbarer Nähe Ressourcen, die in den Flusstälern des Nasca-Beckens sonst nicht vorhanden wären.

Jüngste Forschungen in Palpa ergaben erste Hinweise auf menschliche Präsenz im Archaikum (ab 3500 v. Chr.) sowie eine permanente Besiedlung spätestens seit der Initialperiode (ab 1500 v. Chr. $)^{15}$. Ein starkes Bevölkerungswachstum und eine erste kulturelle Blüte erfolgte zur Zeit des Frühen Horizontes, als die Paracas-Kultur (800-200 v. Chr.) in einem weitläufigen kulturellen Austausch mit anderen Regionen des Andenraumes stand. Nach einer Übergangsphase, der sog. Nasca-Initialzeit (120 v. Chr. - 90 n. Chr.), setzte sich diese Blüte in der Frühen Zwischenperiode mit der Nasca-Kultur (90-620 n. Chr.) fort, die sich durch einige technologische Innovationen auszeichnete und deren 
polychrome Keramik zu den Höhepunkten der Handwerkskunst im vorspanischen Andenraum zählt. Gegen Ende der Nasca-Zeit führte jedoch die fortschreitende Aridisierung zu einer Verlagerung der Bevölkerungsschwerpunkte vom Andenfuß in Richtung Osten, d. h. die Täler aufwärts. Aus dem Mittleren Horizont (620-1000 n. Chr.) sind daher zumindest aus der Region Palpa nur wenige Spuren menschlicher Aktiväten erhalten. Auf diese teilweise Entvölkerung folgte erst in der Späten Zwischenperiode ab ca. 1150 n. Chr. aus den Anden heraus eine Neubesiedlung mit einer weiteren kulturellen Blüte, bevor die Nasca-Region im 15 Jh. zunächst ins Inka-Imperium und dann im 16. Jh. in das spanische Kolonialreich eingegliedert wurde.

\section{Die Geoglyphen}

Die Geoglyphen der Paracas- und Nasca-Zeit in der Umgebung der heutigen Stadt Palpa im nördlichen Teil des Nasca-Beckens wurden zwischen 1997 und 2004 näher untersucht. Palpa liegt in einer weiten Schwemmebene, die von zwei Zuflüssen des Río Grande gebildet wird. Während im Westen eine Hügelkette die Grenze des Beckens markiert, ist Palpa im Norden, Osten und Süden von Fußflächen umgeben. Diese nehmen zwar nicht Ausmaße an wie die ca. $40 \mathrm{~km}$ südlich gelegenen, besser bekannten Pampas von Nasca, bieten jedoch besonders südlich von Palpa viel Raum für die Anlage von Geoglyphen, der auch ausgiebig genutzt wurde. In dieser Umgebung wurden auf einer Fläche von $89 \mathrm{~km}^{2}$ auf der Grundlage von speziell dafür angefertigten Luftbildern mehr als 1500 Geoglyphen photogrammetrisch kartiert. Ein großer Teil der kartierten Geoglyphen wurde sodann im Gelände begangen, beschrieben und archäologisch untersucht. Die Feldarbeiten umfassten die Dokumentation von Spuren menschlicher Aktivitäten und Oberflächenfunden ${ }^{16}$, Magnetfeldmessungen auf ausgewählten Geoglyphen ${ }^{17}$ sowie kleinräumige Ausgrabungen von Steinplattformen auf und neben einigen Geoglyphen ${ }^{18}$. Damit liegt nun erstmals für ein Teilgebiet des Nasca-Beckens eine flächendeckende Kartierung und Dokumentation der Geoglyphen vor. Die Auswertungsarbeiten umfassten die Erstellung einer Typochronologie der Geoglyphen, die Interpretation von Funden und Befunden im Hinblick auf die Rekonstruktion menschlicher Aktivitäten, eine virtuelle 3-D-Modellierung der Geoglyphen und des Geländes, GIS-gestützte räumliche Analysen und schließlich eine kulturgeschichtliche Einordnung und Interpretation ${ }^{19}$.

Die Geoglyphen von Palpa sind einerseits Teil desselben kulturellen Phänomens wie die bekannteren Geoglyphen auf den Pampas von Nasca weiter südlich ${ }^{20}$. Das grund-

\footnotetext{
16 Lambers 2006.

$17 \quad$ Gorka et al. 2007.

18 Reindel et al. 2006.

19 Lambers 2006.

20 Aveni 1990; Reiche 1993.
} 
legende Repertoire an Formen und Typen, die Konstruktionstechniken, die assoziierten Funde und Befunde und damit auch der chronologische Rahmen sind hier wie dort weitgehend gleich. Die Geoglyphen von Palpa weisen jedoch einige lokale Besonderheiten auf. So finden sich hier nur wenige der eingangs erwähnten bekannten Tierfiguren, wie sie vor allem am Nordrand der Pampas von Nasca vorkommen. Dies ermöglichte eine Fokussierung der Untersuchungen auf die viel häufigeren geometrischen Geoglyphen. Wie erst im Laufe der Untersuchungen klar wurde, sind allerdings in Palpa anthropomorphe Figuren, die überwiegend aus frühen Phasen stammen, in größerer Zahl vertreten. Durch die größere zeitliche Tiefe konnten Ursprung und Entwicklungsgeschichte der Geoglyphen in Palpa besonders gut nachvollzogen werden.

\subsection{Biomorphe Geoglyphen der Paracas-Zeit}

Während der überwiegende Teil der Geoglyphen in Palpa zur Nasca-Zeit angelegt wurde $(120$ v. Chr. -620 n. Chr.), sind in den Trockentälern zwischen den Fußflächen auch zahlreiche Geoglyphen aus der Paracas-Zeit erhalten (800-200 v. Chr. $)^{21}$. Die Bedeutung dieser Befundgattung wurde erst im Laufe der Projektarbeiten erkannt, weil die frühen Bodenzeichnungen oftmals in einem schlechten Erhaltungszustand sind. Dies hängt nicht nur mit ihrem höheren Alter, sondern auch mit einer weiteren Eigenschaft zusammen, die für diese Befunde typisch und ein wesentlicher Schlüssel zu ihrem Verständnis ist. So finden sich praktisch alle frühen Geoglyphen in Hanglage (Abb. 2), wo sie stärker als anderswo der natürlichen Erosion ausgesetzt sind - dies im Gegensatz zu späteren Geoglyphen, die zumeist auf den ebenen Fußflächen angelegt wurden. Die Hanglage korrespondiert auch mit einer geringeren Größe der frühen biomorphen Geoglyphen, die zwischen 5 und $30 \mathrm{~m}$ beträgt, im Gegensatz zu teils mehreren Kilometer langen Linien und Trapezen auf den Fußflächen.

Der essentielle Unterschied zu späteren Geoglyphen besteht jedoch in den Motiven. Dargestellt sind - neben einigen Tieren (Vögel, Katzen, Fische) und rituellen Gegenständen (tumi, ein Zeremonialmesser) - anthropomorphe Wesen, teils in Frontalansicht, teils in Seitenansicht, wobei das Gesicht immer dem Betrachter zugewandt ist (Abb. 2). Es handelt sich um mit nur wenigen geräumten Linien und Steinanhäufungen ausgeführte Figuren, die häufig einen auffälligen Kopfputz tragen, manchmal Gegenstände in den Händen halten, gelegentlich jedoch auch nur als Kopf ausgeführt sind. Vergleichbare, oft bis in Details übereinstimmende Figuren sind von Textilien der Paracas-Kultur bekannt, die als Teil von Mumienbündeln an Bestattungsplätzen in der trockenen Küstenwüste erhalten blieben ${ }^{22}$. Dargestellt sind darauf teils Menschen, teils Mischwesen mit 
menschlichen, tierlichen und übernatürlichen Zügen, die aufgrund ihrer Merkmale und Paraphernalia als Ahnen, Priester oder Schamanen oder auch als übernatürliche Kräfte oder Gottheiten gedeutet werden ${ }^{23}$. Bei einer Figur mit auffällig betonten Augen handelt es sich um das sog. Augenwesen (Ser Oculado bzw. Oculate Being), das als wichtigste Figur im Pantheon der Paracas-Kultur gedeutet wird ${ }^{24}$ und als Vorläufer des zur Nasca-Zeit in der Ikonographie häufig vertretenen >Anthropomorphen Mythischen Wesens< gilt (Ser Mítico Antropomorfo bzw. Anthropomorphic Mythical Being oder »AMB«25. Diese Motive wie auch der Grabkontext der Textilien deuten auf einen im weitesten Sinne kultischen Zusammenhang hin.

Vergleichbare Motive finden sich zudem auf Paracas-Keramik sowie in einfacherer Form auf Felsbildern der Paracas-Zeit ${ }^{26}$. Letztere Parallele ist deshalb wichtig, weil sie auf den wahrscheinlichen Ursprung der frühen Geoglyphen hinweist. Die Paracas-Petroglyphen finden sich im Gelände auf einzelnen Felsblöcken oder Felswänden fast immer in Hanglage. Diese topographische Situation entspricht derjenigen der frühen Geoglyphen. Vermutlich entstand die Geoglyphen-Tradition also aus einer Übertragung bekannter Motive, die bereits auf Textilien, Keramik und auch Felsen ausgeführt wurden, auf die steinige Oberfläche des Wüstenbodens. Es ist naheliegend anzunehmen, dass die Kontexte, aus denen diese Motive ursprünglich stammten, auch zur Interpretation der frühen Geoglyphen beitragen können. Zwar handelt es sich um sehr verschiedene Kontexte: im Falle der Textilien um aufwendige, festen Mustern folgende Bestattungszeremonien ${ }^{27}$, im Falle der von $\mathrm{Fux}^{28}$ besprochenen Petroglyphen um Aktivitäten an Rast- und Handelsplätzen entlang von Fernhandelswegen. Diesen Kontexten ist jedoch gemeinsam, dass die Bilder an bestimmten Orten in Relation zu spezifischen, in der Gruppe durchgeführten Handlungen standen und ihnen in diesem Rahmen offensichtlich eine visuelle Bedeutung zukam. Es bestand also jeweils ein Zusammenhang zwischen Bild, Raum und Handlung.

Dies dürfte auch für die frühen Geoglyphen gelten. Ihre Lage im Gelände erlaubte es, sie aus einer gewissen Distanz, z. B. von einer Fußfläche, vom gegenüberliegenden Hang oder auch vom Boden des Trockentales aus, ganz zu überblicken und damit das Motiv zu betrachten (Abb. 2). Es handelte sich also tatsächlich um Bilder, die zur Betrachtung dienten - dies im Gegensatz zu den oben angesprochenen Tierfiguren der Nasca-Zeit, die erst mit heutigen technischen Mitteln zur Gänze betrachtet werden können. Die Parallelen zu ähnlichen anthropomorphen Motiven auf Textilien und Felswänden deuten darauf hin, dass diese Bilder im Zusammenhang mit bestimmten Handlungen ihre Bedeutung erlangten - Handlungen, die aus Gründen der Bildwahrnehmung vermutlich in einiger

23 Makowski 2000; Silverman - Proulx 2002; Proulx 2006.

24 Isla - Reindel 2007.

25 Makowski 2000; Proulx 2006.

26 Fux et al. 2009.

27 Chamorro 2009.

28 Fux et al. 2009. 


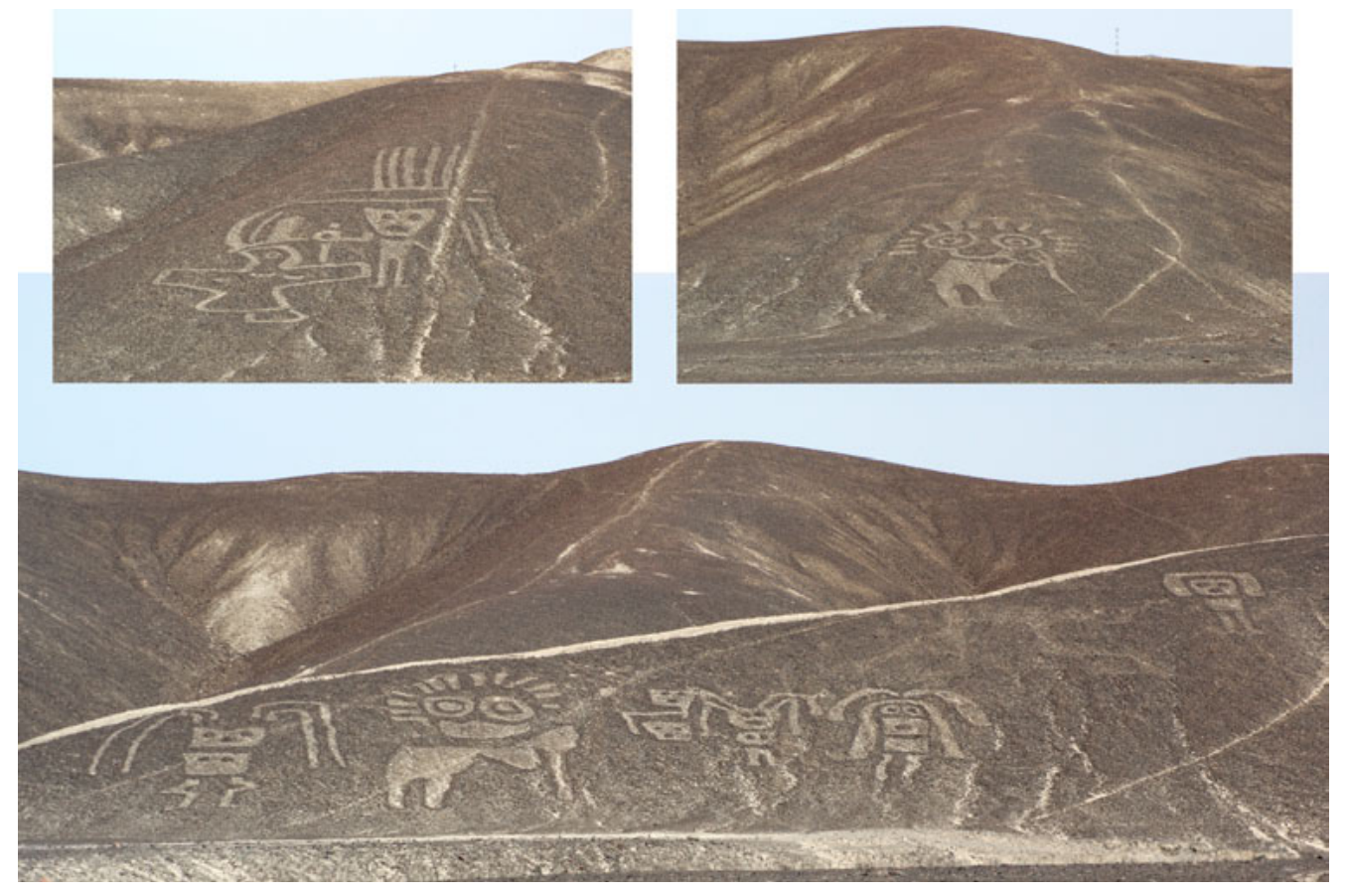

Abb. 2 | Restaurierte anthro-pomorphe Geoglyphen der ParacasZeit im Río Grande-Tal bei Llipata südwestlich von Palpa, Ansicht vom Trockental im Süden aus.

Distanz stattfanden. Somit würde eine Geoglyphe nicht selbst als Ort für Handlungen gedient, sondern im umliegenden Gelände eine potentielle Aktivitätszone aufgespannt haben. Bestätigung findet diese Hypothese in der Tatsache, dass direkt bei und auf den frühen anthropomorphen Geoglyphen kaum Spuren von Aktivitäten festgestellt werden konnten - dies in deutlichem Gegensatz zu den späteren geometrischen Geoglyphen auf den Fußflächen, auf denen eine Vielfalt von Funden und Befunden auf häufige Aktivitäten hindeuten (s. u.). Vergleichbare Konstellationen zwischen Geoglyphen und Aktivitätszonen finden sich auch bei den späteren Geoglyphen der nordchilenischen Atacama-Wüste ${ }^{29}$.

Wie die hier postulierten Handlungen aussahen, zu denen die frühen Geoglyphen in visueller Beziehung standen, ist archäologisch schwer zu fassen. Dies liegt nicht nur daran, dass jeweils größere Zonen dafür in Frage kamen, die Handlungen also an verschiedenen Orten, von denen aus eine visuelle Verbindung zu den Geoglyphen bestand, stattfinden konnten; sondern auch - vor allem - daran, dass die gesamte Umgebung der frühen Geoglyphen zur Nasca-Zeit intensiv und großflächig genutzt und umgestaltet wurde (s. u.), folglich viele Spuren dieser Aktivitäten bereits damals zerstört worden sein dürf-

29 Lambers im Druck. 
ten. Dennoch hat sich südlich von Palpa ein Befund erhalten, der möglicherweise Ausdruck solcher Aktivitäten ist. Auf der Pampa von San Ignacio fanden sich in einer flachen Senke zahlreiche Keramikdeponierungen der Paracas-Zeit. Dabei handelt es sich zumeist um zerbrochene Einzelgefäße der Keramik-Phase Ocucaje 8 (ca. 370-200 v. Chr.), die auf einer Fläche von ca. $50 \times 50 \mathrm{~m}^{2}$ auf dem Wüstenboden deponiert wurden. Aufgrund ihrer Form könnten die Gefäße Speisen und Getränke enthalten haben, jedoch waren davon keine Spuren erhalten. Der zunächst nicht ersichtliche Kontext könnte durch anthropomorphe Paracas-Geoglyphen an einem flachen Hang in geringer Entfernung hergestellt worden sein. Zwar sind diese von der flachen Senke aus nicht direkt sichtbar. Doch die gesamte Umgebung beider Befunde, Keramikdeponierungen und anthropomorphe Geoglyphen, wurde zur Nasca-Zeit in einen der größten Komplexe geometrischer Geoglyphen des gesamten Nasca-Beckens umgestaltet. Wie die Fußfläche zur Paracas-Zeit aussah, ist daher kaum zu rekonstruieren. Es scheint zumindest denkbar, dass noch weitere frühe Geoglyphen und auch Keramikdeponierungszonen existierten, die heute nicht mehr erhalten sind. Somit sind diese Keramikdeponierungen zwar kein Beleg, aber ein Indiz für die vermuteten Handlungen, die in einem visuellen Zusammenhang zu den frühen anthropomorphen Geoglyphen standen.

Während die frühen anthropomorphen Geoglyphen Teil eines ikonographischen Komplexes waren, der auf verschiedenen Medien verwirklicht wurde (Textilien, Keramik, Felsen, Wüstenboden), entwickelte sich aus der Dynamik der Bild-Raum-HandlungsBeziehungen bald ein eigenständiges Phänomen. Dazu trug nicht nur bei, dass Bodenzeichnungen durch Verlagerung der Steine des Wüstenpflasters sehr einfach anzulegen waren, was bald zur Anlage größerer Geoglyphen und zu Experimenten mit neuen Formen führte. Entscheidend dürfte vielmehr gewesen sein, dass die Bevölkerung bereits in der Paracas-Zeit regelmäßig in die Wüste hinausging und an verschiedenen Stellen Handlungen vollzog, bei denen die Geoglyphen eine visuelle Rolle spielten. So wurde der wirtschaftlich bis auf geringe Jagdaktivitäten nicht nutzbare Wüstenraum immer stärker in die kulturelle Domäne der Gesellschaft, deren Lebensgrundlage ansonsten die Täler bildeten, einbezogen, was den Weg für weitere Aktivitäten in der Nasca-Zeit bereitete.

\subsection{Geometrische Geoglyphen der Nasca-Zeit}

In Palpa konnte anhand relativ- und absolutchronologischer Daten nachvollzogen werden, dass auf die frühen biomorphen Geoglyphen an den Hängen bald erste gerade Linien folgten, zunächst ebenfalls an den Hängen und dann erstmals auch auf den darüber liegenden Fußflächen. Diese neue Entwicklung entfaltete sich vollends in der Übergangszeit zur Nasca-Periode (120 v. Chr. - 90 n. Chr.) und mündete in der Frühen Nasca-Zeit (90$325 \mathrm{n}$. Chr.) schließlich in eine große formale Vielfalt an figürlichen und geometrischen Bodenzeichnungen, die nun in erster Linie auf den Fußflächen angelegt wurden und 


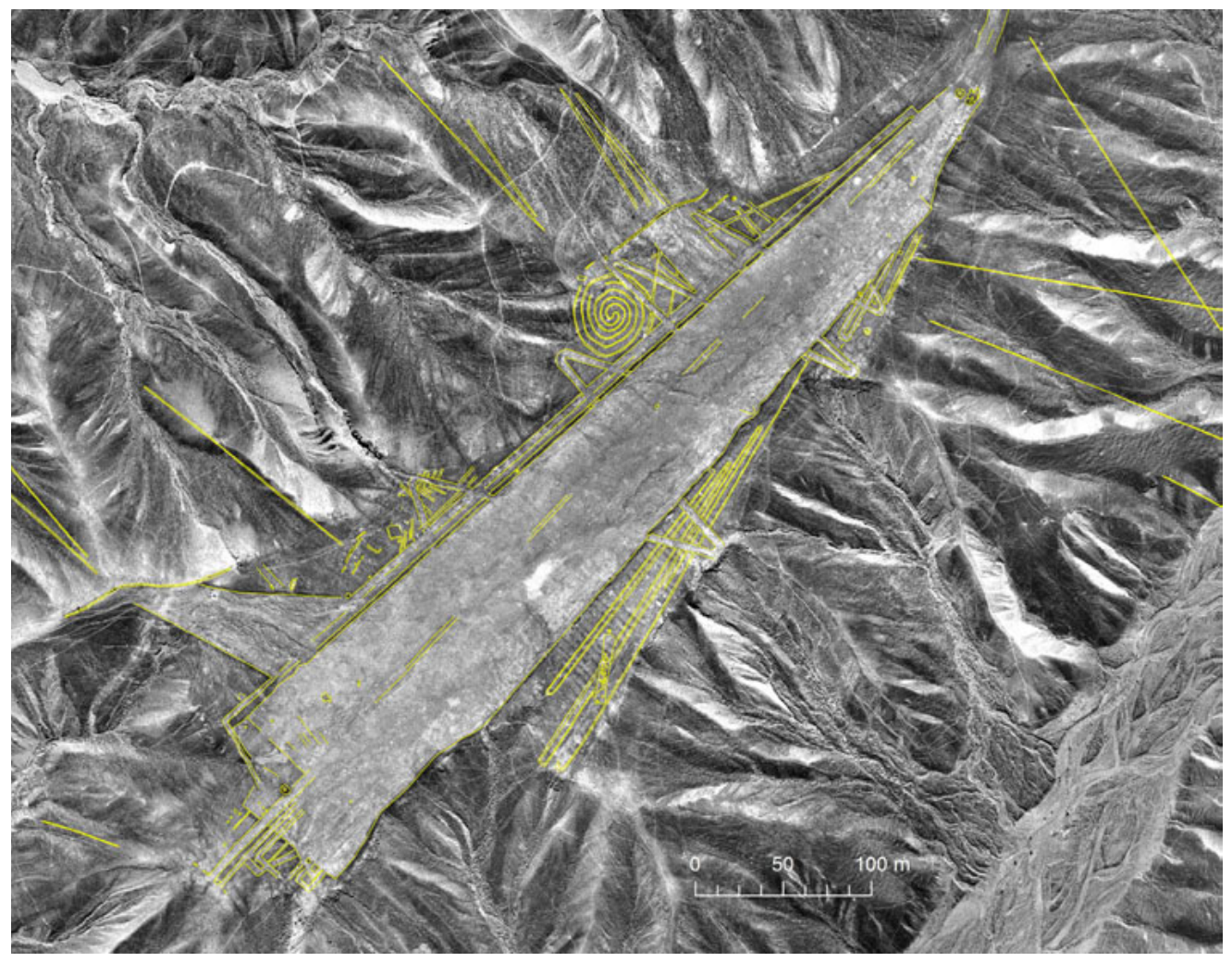

Abb. 3 | Typische Kombination von geometrischen Geoglyphen der Nasca-Zeit auf der Fußfläche oberhalb von Llipata südwestlich von Palpa.

dort Handlungsräume definierten. Spätestens ab dieser Phase waren die Geoglyphen zu einem eigenständigen, von anderen Medien unabhängigen Kulturphänomen geworden, das sich durch eine hohe Dynamik auszeichnete, einen erheblichen Teil der gesellschaftlichen Arbeitsleistung absorbierte, soziale Handlungen und Beziehungen definierte und zu einem Grundpfeiler dessen wurde, was Helaine Silverman treffend als »Nascaness« bezeichnet hat ${ }^{30}$.

Zwar umfasst der sehr vielfältige Komplex von Geoglyphen der frühen Nasca-Zeit in Palpa auch wenige figürliche Geoglyphen, die den bekannten Figuren der Pampas von Nasca ähneln, doch soll es im Folgenden um die geometrischen Geoglyphen gehen, die die große Mehrheit der Geoglyphen bilden. Dabei handelt es sich um Linien - gerade, zickzackförmige, mäandrierende, spiralförmige u. a. - und geräumte Flächen - trapezförmige, rechteckige und unregelmäßige - sowie Kombinationen von beiden (Abb. 3). 
Die Länge der Trapeze reicht von mehreren Metern bis zu knapp $2 \mathrm{~km}$, die Breite der Linien von einer Handbreit bis zu einigen Metern. Das heute sichtbare Endergebnis von mehreren Jahrhunderten der Anlage und Nutzung von Geoglyphen auf den weitläufigen Fußflächen rund um Palpa besteht neben einer Vielzahl einzelner Geoglyphen aus verschiedenen großen Komplexen von zahlreichen sich überlagernden und schneidenden Linien und Flächen (Abb. 3). Auf diesen Geoglyphenkomplexen sind vielfältige Spuren menschlicher Aktivitäten erhalten, die sich auf oder bei den Geoglyphen abspielten ${ }^{11}$ dies in deutlichem Gegensatz $\mathrm{zu}$ den oben beschriebenen frühen anthropomorphen Geoglyphen, bei denen die entsprechenden Aktivitäten vermutlich in einiger Entfernung stattfanden.

Die erste Art von Handlung, die rekonstruiert werden konnte, war die Anlage der Bodenzeichnungen. Dies mag trivial erscheinen, doch wurde auch die Bedeutung dieses Befundes erst im Laufe der Untersuchungen klar. Bisher wurde in der Literatur überwiegend der Nutzung der Geoglyphen Bedeutung beigemessen, während ihre Anlage allenfalls unter technischen Gesichtspunkten von Interesse zu sein schien. Die Befundlage in Palpa deutet jedoch auf eine erhebliche soziale Bedeutung dieses Arbeitsschrittes hin. Diese ergibt sich aus einer über lange Zeiträume fortwährenden Anlage neuer bzw. Umgestaltung vorhandener Geoglyphen, die schließlich zu den heute sichtbaren komplexen Konzentrationen führte. Zahlreiche Überlagerungen sowie unvollendete Geoglyphen erlauben es, diesen Prozess im Detail zu studieren ${ }^{22}$. Teils wurden bei der Anlage neuer oder der Erweiterung bestehender Geoglyphen vorhandene Geoglyphen respektiert oder in das neue Design integriert, teils wurden sie aber auch überlagert und damit obsolet. Linen wurden häufig in Trapeze umgestaltet, Trapeze seitlich erweitert, manche Flächen mehrfach geräumt. Diese Arbeiten wurden in unterschiedlicher Intensität anscheinend über längere Zeiträume hinweg durchgeführt; einige Arbeiten blieben auch unvollendet. Beides stand einer zwischenzeitlichen Nutzung jedoch nicht im Wege, wie Nutzungsspuren auf unvollendeten oder in Umgestaltung befindlichen Geoglyphen deutlich zeigen. Es ging also offensichtlich nicht darum, z. B. ein Trapez einmalig in möglichst effizienter Weise anzulegen, um es dann über lange Zeiträume auf welche Weise auch immer zu nutzen. Vielmehr kam der Anlage selbst bereits eine große Bedeutung als sozialer Akt zu.

Die Details des Arbeitsprozesses deuten darauf hin, dass in größeren Gruppen gearbeitet wurde, vermutlich angeleitet von Spezialisten. Über die Zusammensetzung dieser Gruppen wissen wir nichts. Aufgrund des Umfanges sowie der Häufigkeit und Dauer der Anlage- und Umgestaltungsaktivitäten ist es jedoch wahrscheinlich, dass zeitweilig große Bevölkerungsteile beteiligt waren. In diesem Zusammenhang ist zu bedenken, dass die Nasca-Kultur mit wenigen Ausnahmen kaum monumentale Architektur kannte, wie sie in der zeitgleichen Moche-Kultur an der Nordküste Perus so spektakuläre Gestalt annahm.

$31 \quad$ Lambers 2006; Reindel et al. 2006.

32 Lambers 2006. 


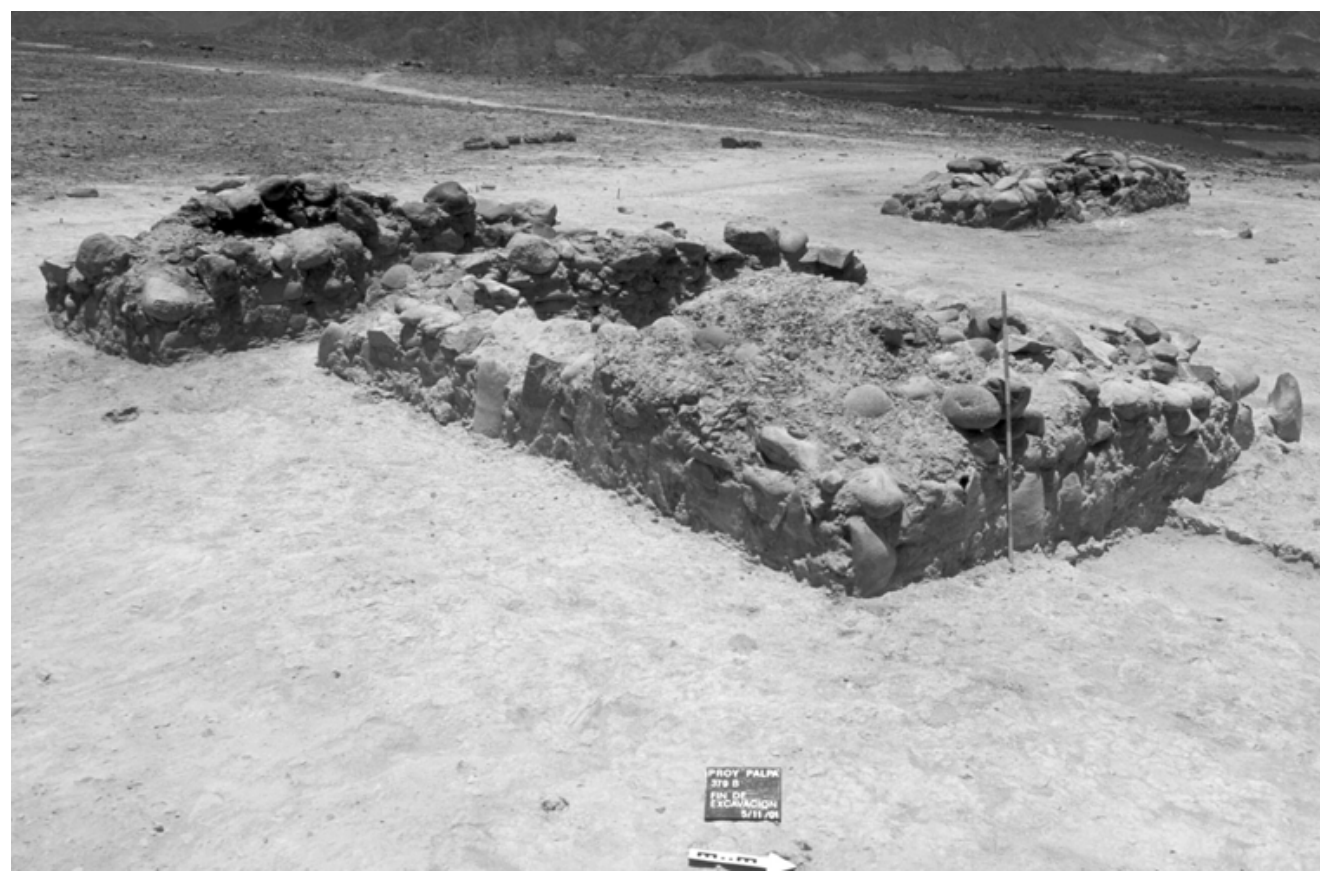

Abb. 4 | Ausgegrabene Steinplattformen am nordöstlichen Ende des zentralen Trapezes des in Abb. 3 wiedergegebenen Geoglyphenkomplexes auf der Fußfläche oberhalb von Llipata südwestlich von Palpa.

Im Nasca-Becken wurde eine vergleichbare Arbeitsleistung stattdessen in die Anlage oder Umgestaltung der Geoglyphen investiert ${ }^{33}$. Dies deutet auf jährlich wiederkehrende, vermutlich vom Zyklus der landwirtschaftlichen Tätigkeiten abhängige Bauarbeiten hin, was auch die häufigen Umgestaltungen erklären würde.

Was sodann gemeinhin unter der Nutzung der Geoglyphen verstanden wird, umfasste nach der Befundlage in Palpa eine Bandbreite verschiedener Tätigkeiten. Am klarsten nachweisen ließen sich ${ }^{34}$ :

- die häufig wiederkehrende Begehung von Linien, in geringerem Maße auch von großen Trapezen, vor allem entlang deren Rändern,

- die Deponierung von Keramikgefäßen, die Speisen oder Getränke enthalten haben könnten, auf oder neben den Rändern von Linien und Trapezen, wobei viele Gefäße offensichtlich intentionell zerschmettert wurden,

- der Bau von Steinplattformen auf Trapezen (Abb. 4) oft lange nach deren ursprünglicher Anlage, teils verbunden mit der Errichtung hoher Holzpfosten, die eventuell zu Orientierungszwecken oder zur Aufhängung von Gegenständen dienten,

34 Lambers 2006; Reindel et al. 2006. 
- die Deponierung von Keramikgefäßen, Textilien, Feldfrüchten, Muscheln und Krebsen auf den Steinplattformen, offenbar in rituellem Kontext.

Daneben ist auf den gesamten Fußflächen ein lockerer Fundschleier aus Keramikscherben, aber auch Textilresten, Steinwerkzeugen etc. festzustellen, der weitere menschliche Präsenz und Aktivität anzeigt. Diese fällt, nach den datierbaren Keramikscherben zu urteilen, chronologisch überwiegend ebenfalls mit der Nutzung der Geoglyphen zusammen und dürfte daher mit ihnen zu tun gehabt haben. Einzige Ausnahme sind Funde aus der Späten Zwischenperiode (ca. 1150-1550 n. Chr.) im Umfeld größerer Ansiedlungen am Andenfuß, die im Zuge einer Wiederbesiedlung der Palpa-Region von Osten her auf den Fußflächen und teils direkt auf den Geoglyphen von einer Bevölkerung angelegt wurden, die die Geoglyphentradition offenbar nicht mehr kannte oder ihr jedenfalls keinen Wert mehr beimaß.

Aus der Nasca-Zeit werden menschliche Aktivitäten am klarsten direkt auf den Geoglyphen fassbar: auf Linien, entlang den Rändern von Trapezen, rund um Steinplattformen auf Trapezen. Die Geoglyphen definierten auf den weiten Hochflächen also Handlungsräume und gaben den dort stattfindenden Tätigkeiten durch ihre Form Orientierung und Ziel, beispielsweise indem eine Linie einen abzuschreitenden Weg vorgab (Abb. 5) oder ein Trapez zu einer Plattform an seinem Ende hinführte. Gleichzeitig entstanden die Geoglyphen selbst durch andere Handlungen - die Anlage und Umgestaltung von Geoglyphen -, was die intensive Wechselwirkung zwischen Raum und Handlung anzeigt. Bild- und Bedeutungsträger waren in diesem Kontext jedoch nicht mehr, wie noch zur ParacasZeit, die Geoglyphen selbst, sondern die Gegenstände, die auf ihnen deponiert wurden, vor allem Keramikgefäße. Die außerordentlich reich verzierten polychromen Keramikgefäße der Nasca-Zeit weisen eine vielfältige Ikonographie auf, die die Lebenswelt der Nasca-Gesellschaft samt ihren natürlichen, sozialen und metaphysischen Elementen eindrucksvoll illustrier ${ }^{35}$. Interessanterweise sind darauf jedoch - mit wenigen unsicheren Ausnahmen ${ }^{36}$ - nirgends Geoglyphen abgebildet, zumindest nicht in einer für uns heute erschließbaren Form. Stattdessen sind Handlungen und Kontexte abgebildet, die möglicherweise auf Geoglyphen stattgefunden haben, so z. B. auf Steinplattformen abgestellte Keramikgefäße oder Holzpfosten mit daran befestigten Bannern und Trophäenköpfen, des Weiteren Schamanen und mythische Wesen, die in kultischen Ritualen eine Rolle gespielt haben dürften und deren Gewänder und Schmuck wiederum die Rolle von Bildträgern einnahmen ${ }^{37}$. Dies bestätigt die Hypothese, dass die geometrischen Geoglyphen zur Nasca-Zeit nicht mehr selbst als Bildträger dienten, sondern den räumlichen Rahmen für Handlungen definierten, die dann wiederum auf anderen Medien abgebildet wurden.

37 Silverman - Proulx 2002. 


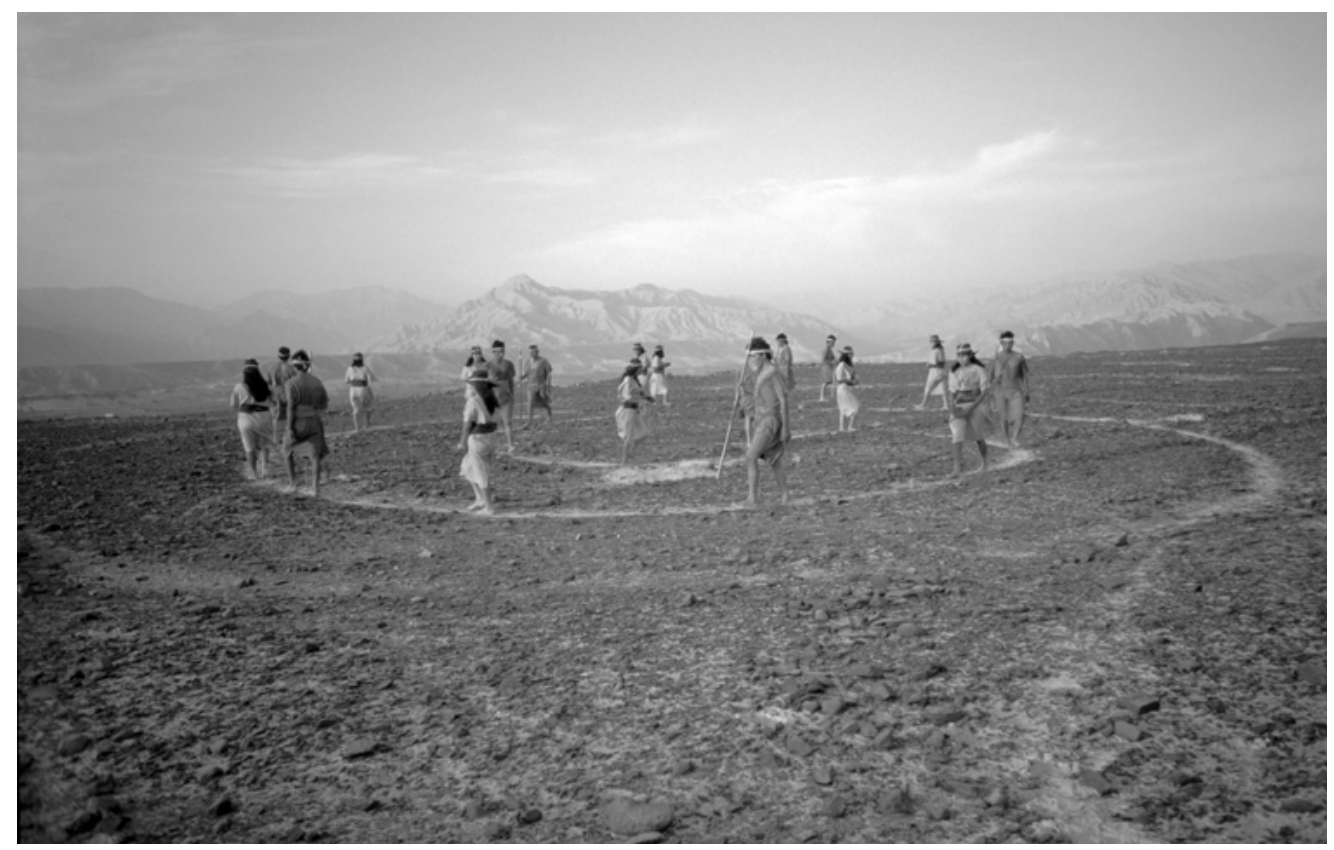

Abb. 5 | Nachgestellte Prozession auf einer Spirale des in Abb. 3 gezeigten Fundortes oberhalb von Llipata südwestlich von Palpa.

Über Inhalt, Verständnis und Bedeutung dieser Handlungen lassen sich nur recht allgemeine Hinweise aus dem archäologischen Befund ablesen. Zum einen haben die Gegenstände, die auf den Steinplattformen deponiert wurden, alle im weitesten Sinne einen Zusammenhang mit Wasser ${ }^{38}$ : die Feldfrüchte, deren Ernte vom Bewässerungsfeldbau abhing, die Flusskrebse, die aus den nur zur Regenzeit in den Bergen Wasser führenden Flüssen stammten, und nicht zuletzt die Spondylusmuscheln. Deren Wanderungsbewegungen entlang der Küste in Abhängigkeit von El-Niño-Ereignissen, welche die Verbreitung von Regenfällen an Land beeinflussen, waren im gesamten Andenraum bekannt und ließen Spondylusmuscheln zu einem wichtigen Kultgegenstand werden. Die Deponierung von Objekten, die thematisch Bezüge zu Wasser, Regen und Fruchtbarkeit erkennen lassen, auf Steinplattformen auf Trapezen dürfte im Zusammenhang mit der zunehmenden Aridisierung der Nasca-Region zur Nasca-Zeit ${ }^{39}$ stehen, die zumindest im Raum Palpa am Übergang zum Mittleren Horizont (700-800 n. Chr.) zu einer weitgehenden Abwanderung der Bevölkerung gen Osten führte und die Aufgabe der Geoglyphentradition nach sich zog. Dazu passt, dass mehrere der Steinplattformen, 
auf denen sich die genannten Objekte fanden, deutlich nach der Anlage der Geoglyphen, auf denen sie stehen, errichtet wurden, also in einer späten Phase, als die Aridisierung zunehmend die Lebensgrundlage der Bevölkerung beeinträchtigte.

Zum anderen dürften die Handlungen, die auf den Geoglyphen vollzogen wurden, abgesehen von ihrem vordergründigen Inhalt auch eine erhebliche soziale Bedeutung gehabt haben. Aus der großen Zahl der weit über die Wüstenlandschaft verstreuten Geoglyphen stechen mehrere große Komplexe heraus, die über viele Jahrhunderte genutzt und immer wieder umgestaltet wurden. Ein Vergleich der räumlichen Entwicklung dieser Komplexe mit der Entwicklung der Siedlungsmuster während der Nasca-Zeit zeigt, dass kein direkter Zusammenhang zwischen Geoglyphenkomplexen und bestimmten Siedlungen bestand ${ }^{40}$. Dies bedeutet, dass bestimmte Geoglyphenkomplexe nicht einfach bestimmten Siedlungen zugeordnet waren. Vielmehr war das Siedlungsmuster viel volatiler als das Verbreitungsmuster der Geoglyphen. In den Tälern lösten zahlreiche Kleinsiedlungen, die oft nur über wenige Generationen genutzt wurden, einander ab und folgten dabei der lokalen Wasserverfügbarkeit, was im Laufe der Zeit zu deutlichen Verlagerungen der Siedlungsschwerpunkte führte. Die großen Geoglyphenkomplexe auf den Fußflächen waren dagegen ortsfest; sie bestanden über Jahrhunderte und wurden auch dann weiter genutzt, wenn die nächstgelegenen Siedlungen aufgegeben wurden. Die Menschen, die auf den Geoglyphenkomplexen zusammentrafen, um dort neue Geoglyphen anzulegen oder rituelle Handlungen zu vollziehen, kamen also aus verschiedenen Siedlungen, die zudem im Laufe der Zeit verlagert wurden. Somit handelte es sich um soziale Gruppen, die auf einer gesellschaftlichen Ebene oberhalb der Siedlungen organisiert waren und auch bei Veränderungen im Siedlungsmuster Bestand hatten.

In diesem Zusammenhang erscheint es interessant, dass ein wichtiges Kriterium für die Auswahl eines Geländes zur Anlage neuer Geoglyphen seine gute Einsehbarkeit war, wie GIS-basierte Sichtbarkeitsstudien zeigten ${ }^{41}$. Wie oben dargelegt, ging es dabei nicht um die Sichtbarkeit der Geoglyphen selbst, die ja nur den Rahmen für darauf zu vollziehende Handlungen definierten. Vielmehr war der Punkt wesentlich, dass diese Handlungen und die daran beteiligten Personen gesehen werden konnten - vom umliegenden Gelände, von anderen Geoglyphenkomplexen und selbst von den Siedlungen im Tal aus. Offensichtlich hatten diese Handlungen und Rituale einen öffentlichen Charakter, weshalb man die Geoglyphen auch als >Bühnen $<$ mit darauf agierenden Akteuren verstehen kann. Da gleichzeitig mehrere große Komplexe in Benutzung standen, die, wie oben dargelegt, mit größeren sozialen Gruppen oberhalb der Ebene einzelner Siedlungen assoziiert gewesen sein dürften, könnten diese von allen sichtbaren Handlungen einen kompetitiven Charakter gehabt haben, indem die verschiedenen sozialen Gruppen ihre Stellung in der Nasca-Gesellschaft verhandelten und definierten. Dazu passt die Beob-

$40 \quad$ Lambers 2006.

41 Lambers - Sauerbier 2009 . 
achtung, dass sich das Geoglyphenrepertoire ab der mittleren Nasca-Zeit auf immer weniger standartisierte Typen (vor allem Trapeze und Linien) reduzierte, gleichzeitig jedoch immer größere Geoglyphen angelegt wurden. Dies könnte damit erklärt werden, dass bei der Anlage neuer Geoglyphen um gesellschaftliche Akzeptanz gerungen wurde, was die Hypothese vom kompetitiven Charakter unterstützt. Grundsätzlich sind Fragen zur Sozialstruktur der Nasca-Gesellschaft und ihres Wandels im Laufe der Zeit aufgrund der Befunde von den Geoglyphen allein jedoch kaum zu beantworten. Unzweifelhaft ist allerdings, dass die Aktivitäten, die mit den Geoglyphen im Zusammenhang standen, eine erhebliche soziale Bedeutung für die Nasca-Gesellschaft hatten.

\section{Von Bildern zu Bühnen}

Obwohl keine Überlieferungen zur konkreten Bedeutung und Funktion der Paracas- und Nasca-zeitlichen Geoglyphen existieren, lassen sich aus ihrem archäologischen Kontext weitreichende Schlüsse ziehen. Simple und eher plakative Bildansprachen, wie sie lange Zeit üblich waren und noch heute oft den Anforderungen des Tourismus geschuldet sind, greifen für die Erklärung des Geoglyphenphänomens zu kurz. Aus dem komplexen Wechselspiel zwischen Geoglyphen, Naturraum und menschlichen Aktivitäten ergeben sich jedoch umfassende Implikationen für die soziale und kulturelle Rolle der Geoglyphen.

Die ersten Geoglyphen der Paracas-Zeit waren Teil eines ikonographischen Komplexes, der sich über verschiedene Medien erstreckte: Keramik, Textilien, Felsen, Wüstenboden. Es handelte sich um biomorphe Abbildungen mit teils übernatürlichen Zügen. Ihr jeweiliger Kontext auf verschiedenen Medien weist darauf hin, dass es sich tatsächlich um Bilder handelte, die aus einer gewissen Distanz betrachtet und gedeutet wurden. Ihre visuelle Bedeutung erlangten sie wahrscheinlich im Rahmen verschiedener Handlungen, die ihre Betrachter in ihrem Blickfeld ausführten. Die Geoglyphen der Paracas-Zeit definierten also Handlungsräume in der Wüste.

Aus den Anfängen einer medienübergreifenden Ikonographie entwickelte sich am Übergang zur Nasca-Zeit ein eigenständiges Phänomen, in dem die Geoglyphen sich von Bildern zu >Bühnen< wandelten. Die assoziierten Handlungen fanden nun nicht mehr bei, sondern auf den Geoglyphen statt; die Handlungsräume wurden nun nicht mehr visuell aufgespannt, sondern physisch markiert. Die Geoglyphen büßten dabei ihre bildliche Bedeutung ein; vielmehr waren es nun die Handlungen, die auf den Geoglyphen stattfanden, und die daran beteiligten Akteure, die gesehen werden sollten und die wahrscheinlich auch dargestellt wurden, z. B. auf Keramikgefäßen und Textilien. Aus den Bildern waren also Bühnen für Handlungen geworden, denen eine wichtige rituelle und soziale Bedeutung zukam.

Die Geoglyphen von Palpa und Nasca sind damit ein Beispiel dafür, wie Menschen ihre Umwelt mittels ihrer Handlungen sozial und kulturell durchdringen und gestalten. 
Obwohl der Naturraum in der Wüste am Fuße der Anden an sich bereits spektakulär ist, tritt er in seiner Bedeutung hinter dem von Menschen definierten sozialen Raum und seiner physischen Manifestation, den Geoglyphen, zurück. Im Sinne der Definition von Knapp und Ashmore ${ }^{42}$ handelt es sich sowohl um eine »constructed « als auch um eine »conceptualized « Landschaft, in der sich kulturelle Konzepte mittels physischer Modifikationen in der Landschaft manifestierten und Orte schufen, die mit bestimmten Handlungen und Bedeutungen assoziiert wurden.

Wenn heutige Besucher der Region die Geoglyphen aus der Luft betrachten, nehmen sie nicht nur eine ahistorische Perspektive ein, sondern müssen auch auf zentrale Elemente zum Verständnis der Geoglyphen verzichten: die Menschen, die sich zur Paracasund Nasca-Zeit auf und bei den Geoglyphen versammelten, sowie die kulturell und sozial eingebetteten Handlungen, die sie dort durchführten. Nur unter Berücksichtigung dieses Kontextes erschließen sich Funktion und Bedeutung der Geoglyphen von Palpa und Nasca. 


\section{Bibliographie}

\section{Aveni 1990}

A. F. Aveni (Hg.), The Lines of Nazca (Philadelphia 1990).

\section{Aveni 2000}

A. F. Aveni, Between the Lines: the Mystery of the Giant Ground Drawings of Ancient Nasca, Peru (Austin 2000).

\section{Chamorro 2009}

A. Chamorro Malagón (Hg.), Mantos para la eternidad: textiles Paracas del antiguo Perú (Madrid 2009).

\section{Clados 2006}

C. Clados, Neue Erkenntnisse zur Ikonographie der Geoglyphen von Nazca, Ethnographisch-Archäologische Zeitschrift (EAZ) 47, 2006, 299-324.

\section{David - Thomas 2008}

B. David - J. Thomas, Landscape Archaeology: Introduction, in: B. David - J. Thomas (Hgg.), Handbook of Landscape Archaeology (Walnut Creek 2008) 27-43.

Eitel et al. 2005

B. Eitel - S. Hecht - B. Mächtle - G. Schukraft - A. Kadereit - G. A. Wagner - B. Kromer I. Unkel - M. Reindel, Geoarchaeological Evidence from Desert Loess in the Nazca-Palpa Region, Southern Peru: Paleoenvironmental Changes and Their Impact on Pre-Columbian Cultures, Archaeometry 47, 2005, 137-158.

Eitel - Mächtle 2009

B. Eitel - B. Mächtle, Man and Environment in the Eastern Atacama Desert (Southern Peru): Holocene Climate Changes and Their Impact on Pre-Columbian Cultures, in: M. Reindel - G. A. Wagner (Hgg.), New Technologies for Archaeology: Multidisciplinary Investigations in Palpa and Nasca, Peru (Heidelberg 2009) 17-37.

\section{Fux et al. 2009}

P. Fux - M. Sauerbier - T. Kersten - M. Lindstädt - H. Eisenbeiß, Perspectives and Contrasts: Documentation and Interpretation of the Petroglyphs of Chichictara, Using Terrestrial Laser Scanning and Image-Based 3D Modeling, in: M. Reindel - G. A. Wagner (Hgg.), New Technologies for Archaeology: Multidisciplinary Investigations in Palpa and Nasca, Peru (Heidelberg 2009) 359-377.

\section{Gorka et al. 2007}

T. Gorka - J. W. E. Fassbinder - K. Lambers, Magnetometry on the Geoglyphs of Palpa and Nasca (Peru), Študijné Zvesti 41, 2007, 176-179. 


\section{Gramsch 2003}

A. Gramsch, Landschaftsarchäologie - ein fachgeschichtlicher Überblick und ein theoretisches Konzept, in: J. Kunow - J. Müller (Hgg.), Landschaftsarchäologie und geographische Informationssysteme (Wünsdorf 2003) 35-54.

\section{Horkheimer 1947}

H. Horkheimer, Las plazoletas, rayas y figuras prehispánicas en las pampas y crestas de la Hoya del Río Grande, Revista de la Universidad Nacional de Trujillo II(1), 1947, 45-63.

\section{Isbell 1978}

W. H. Isbell, The Prehistoric Ground Drawings of Peru, Scientific American 239, 1978, 114-122.

\section{Isla 2010}

J. Isla, Perspectivas sobre el proceso cultural en los valles de Palpa, costa sur del Perú, in:

L. Valle Alvarez (Hg.), Arqueología y desarollo, experiencias y posibilidades en el Perú (Lima 2010) 15-52.

\section{Isla - Reindel 2007}

J. Isla - M. Reindel, Los Paracas del sur: una nueva perspectiva desde los valles de Palpa, in: M. Frame - J. Isla - E. Leon et al., Hilos del pasado: el aporte francés al legado Paracas (Lima 2007) 79-91.

\section{Knapp - Ashmore 1999}

A. B. Knapp - W. Ashmore, Archaeological Landscapes: Constructed, Conceptualized, Ideational, in: W. Ashmore - A. B. Knapp (Hgg.), Archaeologies of Landscape:

Contemporary Perspectives (Oxford 1999) 1-30.

\section{Kosok - Reiche I947}

P. Kosok - M. Reiche, The Mysterious Markings of Nazca, Natural History LVI, 1947, 200-207. 237-238.

\section{Lambers 2006}

K. Lambers, The Geoglyphs of Palpa, Peru: Documentation, Analysis, and Interpretation, FAAK 2 (Aichwald 2006).

\section{Lambers im Druck}

K. Lambers, Walking and Marking the Desert: Geoglyphs in South America, in: M. Aldenderfer - J. L. Lanata (Hgg.), The Oxford Handbook of South American Archaeology (New York, im Druck). 


\section{Lambers - Sauerbier 2009}

K. Lambers - M. Sauerbier, Context Matters: GIS-Based Spatial Analysis of the Nasca Geoglyphs of Palpa, in: M. Reindel - G. A. Wagner (Hgg.), New Technologies for Archaeology: Multidisciplinary Investigations in Palpa and Nasca, Peru (Heidelberg 2009) 321-338.

\section{Llobera 1996}

M. Llobera, Exploring the Topography of Mind: GIS, Social Space and Archaeology, Antiquity 70, 1996, 612-622.

\section{Mächtle 2007}

B. Mächtle, Geomorphologisch-bodenkundliche Untersuchungen zur Rekonstruktion der holozänen Umweltgeschichte in der nördlichen Atacama im Raum Palpa / Südperu (Heidelberg 2007).

\section{Makowski 2000}

M. Makowski, Los seres sobrenaturales en la iconografía Paracas y Nasca, in:

K. Makowski - K. M. Hanula et al. (Hgg.), Los dioses del antiguo Perú (Lima 2000) 277-323.

\section{Proulx 2006}

D. A. Proulx, A Sourcebook of Nasca Ceramic Iconography: Reading a Culture through Its Art (Iowa City 2006).

\section{Reiche 1949}

M. Reiche, Los dibujos gigantescos en el suelo de las pampas de Nazca y Palpa: descripción y ensayo de interpretación (Lima 1949).

\section{Reiche 1993}

M. Reiche, Contribuciones a la geometría y astronomía en el antiguo Perú (Lima 1993).

\section{Reindel 2009}

M. Reindel, Life at the Edge of the Desert - Archaeological Reconstruction of the Settlement History in the Valleys of Palpa, Peru, in: M. Reindel - G. A. Wagner (Hgg.), New Technologies for Archaeology: Multidisciplinary Investigations in Palpa and Nasca, Peru (Heidelberg 2009) 439-461.

\section{Reindel et al. 2006}

M. Reindel - J. Isla - K. Lambers, Las estructuras de piedra sobre los geoglifos Nasca en Palpa, Arqueología y Sociedad 17, 2006, 179-222.

\section{Reindel - Wagner 2009}

M. Reindel - G. A. Wagner (Hgg.), New Technologies for Archaeology: Multidisciplinary Investigations in Palpa and Nasca, Peru (Heidelberg 2009). 


\section{Silverman 2002}

H. Silverman, Nasca Settlement and Society on the Hundredth Anniversary of Uhle's Discovery of the Nasca Style, in: W. H. Isbell - H. Silverman (Hgg.), Andean Archaeology I: Variations in Sociopolicital Organization (New York 2002) 121-158.

\section{Silverman - Proulx 2002}

H. Silverman - D. A. Proulx, The Nasca (Oxford 2002).

\section{Abbildungsnachweis}

Abb. 1: Karte H. P. Wittersheim; Abb. 2: Photographie J. Isla; Abb. 3: Karte K. Lambers, Orthophoto IGP, ETH Zürich; Abb. 4: Photographie J. Isla; Abb. 5: Photographie M. Reindel. 